\title{
Metabolic syndrome is associated with reduced flow mediated dilation independent of obesity status
}

\author{
Victoria S Sprung 1,2,3,*, Kelly A Bowden Davies 10 1,3,4,*, Juliette A Norman ${ }^{1,3}$, Andrew Thompson ${ }^{5}$,

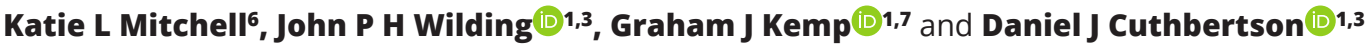

${ }^{1}$ Institute of Life Course and Medical Sciences, University of Liverpool, Liverpool, UK, ${ }^{2}$ Research Institute for Sport and Exercise Sciences, Liverpool John Moores University, Liverpool, UK, ${ }^{3}$ Obesity and Endocrinology Research Group, Liverpool University Hospitals NHS Foundation Trust, Liverpool, UK, ${ }^{4}$ Department of Sport and Exercise Sciences, Manchester Metropolitan University, Manchester, UK, 5 Institute of Systems, Molecular and Integrative Biology, University of Liverpool, Liverpool, UK Institute of Psychology Health and Society, University of Liverpool, Liverpool, UK, and ${ }^{7}$ Liverpool Magnetic Resonance Imaging Centre (LiMRIC), University of Liverpool, Liverpool, UK *(V S Sprung and K A Bowden Davies contributed equally to this work)

Correspondence should be addressed to V S Sprung Email V.S.Sprung@ljmu.ac.uk

\begin{abstract}
Background: Data suggest that metabolic health status, incorporating components of metabolic syndrome (MetS), predicts cardiovascular disease (CVD) risk better than BMI. This study explored the association of MetS and obesity with endothelial function, a prognostic risk factor for incident CVD.

Methods: Forty-four participants were phenotyped according to BMI as non-obese vs obese $\left(<30 \mathrm{or}>30 \mathrm{~kg} / \mathrm{m}^{2}\right)$ and according to the International Diabetes Federation criteria of MetS: $\leq 2$ criteria MetS (MetS-) vs $\geq 3$ criteria MetS (MetS+); (1.) non-obese MetS-vs (2.) non-obese MetS+ and (3.) obese MetS- vs (4.) obese MetS+. Flow-mediated dilation (FMD), body composition including liver fat (MRI and spectroscopy), dietary intake, intensities of habitual physical activity and cardio-respiratory fitness were determined. Variables were analysed using a one-factor betweengroups ANOVA and linear regression; mean $(95 \% \mathrm{Cl})$ are presented.

Results: Individuals with MetS+ displayed lower FMD than those with MetS-. For non-obese individuals mean difference between MetS+ and MetS- was 4.1\% ((1.0, 7.3); $P=0.004)$ and obese individuals had a mean difference between MetS+ and MetS- of $6.2 \%((3.1,9.2) ; P<0.001)$. Although there was no association between BMI and FMD $(P=0.27)$, an increased number of MetS components was associated with a lower FMD $(P=0.005)$, and after adjustment for age and sex, $19.7 \%$ of the variance of FMD was explained by MetS, whereas only $1.1 \%$ was explained by BMI.

Conclusions: In this study cohort, components of MetS, rather than obesity per se, contribute to reduced FMD, which suggests a reduced bioavailability of nitric oxide and thus increased risk of CVD.
\end{abstract}

\section{Introduction}

Obesity is strongly linked with an adverse cardio-metabolic profile and a number of chronic diseases including type 2 diabetes (T2D) and cardiovascular disease (CVD) (1, 2). BMI is widely used clinically to determine the risk of complications relating to an excess accumulation of fat: the higher an individual's BMI, the greater their risk of obesity-related complications (3). In contrast, some data suggest that adults with a higher BMI can have a reduced mortality risk compared to non-obese counterparts, an puzzling finding known as the 'obesity paradox', shown in T2D (4) and CVD (5). Metabolic syndrome (MetS) is defined as a cluster of risk factors including abdominal

Published by Bioscientifica Ltd. 
obesity, hypertension, dyslipidemia and insulin resistance. The International Diabetes Federation (IDF) report the role of MetS in the CVD epidemic and highlight the importance of understanding the further role of vascular regulation and body fat distribution (6).

While obesity also has mechanical and psychological implications, there is a growing recognition that not all obese individuals are 'unhealthy' and not all non-obese individuals are 'healthy', with respect to their metabolic profiles. Some data suggest there is a lower T2D/CVD risk in overweight/obese people when there is an absence of Mets components but that there is a higher T2D/CVD risk in normal weight people in the presence of one/more MetS components (7). This has led to the identification of sub-phenotypes within BMI (i.e. metabolically healthy vs unhealthy obesity and healthy vs unhealthy normal weight), categories determined by the presence/absence of components of the MetS. There is currently no consensus on a precise definition for these terms/BMI subphenotypes with researchers questioning the degree of cardiovascular protection conferred by being metabolically healthy and many suggesting that metabolically healthy obesity represents a 'transient metabolic state' in a progressive and inevitable journey towards T2D and CVD $(8,9,10,11)$.

When considering cardiovascular risk in these metabolically phenotyped groups, previous research has largely focused on the overall incidence of $\operatorname{CVD}(8,9,12$, $13,14)$. While this is important, endothelial function, an early, prognostic and reversible marker of CVD, is much less explored. The endothelium plays a pivotal role in vascular homeostasis (15), and brachial artery flow-mediated dilation (FMD) is predictive of future CVD risk (16). Endothelial dysfunction, characterised by decreased nitric oxide (NO) bioavailability, resulting in vascular inflammation, vasoconstriction, and thrombosis $(17,18)$, has been mechanistically related to the greater risk of cardiovascular events in people with obesity (19, 20). To put this measurement into a pathophysiological perspective, a meta-analysis reports that a $1 \%$ increase in FMD is associated with a pooled relative risk reduction in CVD of 0.87 (95\% CI, 0.83-0.91) (21). Furthermore, there is evidence that FMD has independent prognostic value to predict cardiovascular events that may better that of traditional risk factors (16). Evidence is lacking on how MetS alone, or in combination with obesity, affects FMD.

The aim of this cross-sectional study was to explore the impact of obesity and MetS on endothelial function using measurements of FMD. Careful phenotypic characterisation of participants was undertaken incorporating assessments of lifestyle (including dietary records and physical activity by objective monitoring), measurements of cardio-respiratory fitness (CRF; by $\dot{\mathrm{VO}}_{2}$ ), obesity and body composition (liver fat determined by MR scanning) and of cardio-metabolic health (including assessment of MetS using International Diabetes Federation criteria).

\section{Subjects and methods}

\section{Participants}

Forty-four individuals (30 male, 14 female) with a mean age of $46 \pm 11$ years were recruited via local advertisement across hospital departments and university campuses. Exclusions included cardiovascular, respiratory, kidney, liver and/or endocrine complications, smoking and $>14$ units/week of alcohol consumption; all participants were medication free. The study conformed to the Declaration of Helsinki and was approved by the North West Research Ethics Committee (14/NW/1145; 14/NW/1147; $15 / \mathrm{NW} / 0550)$. All participants were informed of the protocol verbally and in writing before providing written informed consent prior to any assessments.

\section{Study design}

All participants completed habitual monitoring of physical activity (PA) and dietary consumption over a period of 4 days (including 1 weekend day), followed by two assessment visits. The first assessment visit, at Aintree University Hospital, comprised anthropometry, fasting biochemistry, and cardio-respiratory fitness ( $\mathrm{V}_{2}$ peak). The second assessment at the University of Liverpool MRI Centre (LiMRiC) comprised flow mediated dilation (FMD) and proton magnetic resonance spectroscopy ( $\left.{ }^{1} \mathrm{H}-\mathrm{MRS}\right)$. Prior to each study visit, participants were required to fast overnight for $>8 \mathrm{~h}$, abstain from alcohol and caffeine for $24 \mathrm{~h}$ and from exercise for $48 \mathrm{~h}$; up to $500 \mathrm{~mL}$ of water was permitted in the morning of a visit.

\section{Brachial artery flow mediated dilation (FMD)}

Endothelial function was assessed by measuring FMD in response to a $5 \mathrm{~min}$ ischaemic stimulus, induced by forearm cuff inflation placed immediately distal to the olecranon process, as previously described (22). Briefly, baseline images were recorded for $1 \mathrm{~min}$ prior to forearm cuff inflation ( $220 \mathrm{mmHg})$ for $5 \mathrm{~min}$. Artery diameter 
and blood flow velocity recordings resumed $30 \mathrm{~s}$ prior to cuff deflation and continued for 3 min thereafter. Peak brachial artery diameter and blood flow velocity and the time taken to reach these peaks following cuff release were recorded. Post-test analysis of brachial artery diameter was undertaken using custom-designed automated edgedetection and wall-tracking software.

\section{Cardio-respiratory fitness}

$\mathrm{V}_{2}$ peak was determined using the modified Bruce protocol on a treadmill (Model 77OCE, RAM Medisoft Group, Manchester, UK) with breath-by-breath monitoring and analysis of expiratory gases and ventilation (Love Medical Cardiopulmonary Diagnostics, Manchester, UK). The $\dot{\mathrm{V}}_{2}$ peak was determined by any of the following: respiratory exchange ratio $>1.15$, heart rate $>90 \%$ predicted maximum, plateau in $\dot{\mathrm{VO}}_{2}$, or exhaustion; data are presented relative to total body mass and lean mass determined by BIA.

\section{Biochemical measures}

Blood samples were collected and analysed using the Olympus AU2700 analyser (Beckman Coulter, High Wycombe, UK) with standard proprietary reagents as follows: glucose with hexokinase, total cholesterol and HDL-cholesterol with cholesterol esterase/oxidase and triglyceride with glycerol kinase. LDL-cholesterol was calculated according to the Friedewald formula.

\section{Anthropometric measures}

Height was measured while participants were standing upright, with their back and head straight so that their Frankfurt plane was horizontal, to the nearest $0.5 \mathrm{~cm}$ using a stadiometer (Model 220, Seca, Germany). Waist circumference measurements (at the umbilicus) and hip circumference measurements (at the greater trochanter) were taken in duplicate. After $5 \mathrm{~min}$ rest, blood pressure was determined as an average of three measurements using an automated monitor (Dinamap, G \& E Medical, USA). Bio-impedance (BIA; Tanita, BC 420, Dolby Medical Stirling, UK) was used in all participants to quantify body composition; those who were safe for MR scanning had the more detailed measures outlined in the subsequent section.

\section{MR determination of adipose tissue and liver fat}

Magnetic resonance methods were performed using a 1.5 T Siemens Symphony MRI scanner (Siemens Medical
Solutions, Erlangen, Germany) as previously described $(23,24,25)$. Volumetric analysis of adipose tissue was used to quantify regional fat; proton magnetic resonance spectroscopy $\left({ }^{1} \mathrm{H}-\mathrm{MRS}\right)$ was used to determine intrahepatic cellular lipid (IHCL): 'liver fat' percentage relative to water.

\section{Habitual physical activity monitoring and dietary analysis}

Physical activity monitoring

PA was monitored using a validated (26) SenseWear mini armband (BodyMedia Inc., Pittsburgh, PA, USA). Participants were requested to wear the armband at all possible times (except when bathing and swimming (27)), and wear time (recorded as $~ 98 \%$ ) was monitored using SenseWear Professional software (version 8.0). Data collected from the armband included: daily average step count, total energy expenditure, active energy expenditure and time spent in different intensity levels of PA including: sleep, lying down, sedentary, light, moderate, vigorous and very vigorous $(<1.5,>1.5-3$, $>3-6,>6-9,>9$ metabolic equivalents, respectively).

\section{Dietary analysis}

Total energy consumption, carbohydrate, protein and fat content were determined from dietary records by a registered nutritionist (KLM) using Nutritics (Nutrition Analysis Software for Professionals; https://www.nutritics. com/p/home; accessed 17/07/2017).

\section{Individual phenotyping}

Following physiological assessment, participants were phenotyped according to obesity status and presence or absence of MetS. Individuals were characterised into one of four groups based on BMI (non-obese $<30$ vs obese $\geq 30$ $\mathrm{kg} / \mathrm{m}^{2}$ ) and the presence or absence of MetS according to IDF criteria (6); we refer to these groups as (1.) 'non-obese MetS-', (2.) 'non-obese MetS+', (3.) 'obese MetS-' and (4.) 'obese MetS+.

\section{Sample size calculation}

The primary outcome variable was FMD. Based on previous data (22) and a two-sample t-test (post-hoc comparison) with a 0.05 two-sided significance level, a sample size of ten per group would have $80 \%$ power to detect a difference in means of $3.5 \%$, assuming a common S.D. of $2.5 \%$ (G*Power 3.1 .5 (28)). 


\section{Statistical analysis}

All data were explored for normality by visual inspection. Comparisons of group demographics were explored using one factor between-groups ANOVA for continuous variables and chi-squared for categorical outcomes. The main outcome variables (e.g. FMD, cardio-respiratory fitness, and liver fat) were analysed using a one factor between-groups ANOVA, with Bonferroni correction for multiple comparisons. All FMD data were analysed and are presented as covariate-controlled for baseline artery diameter measured prior to the introduction of hyperaemia in each test; this approach is more accurate for scaling changes in artery diameter than simple percentage change $(29,30)$. Regression models, adjusted for age and sex, were fitted to categories of BMI and number of MetS components to explore the association with FMD. Finally, we estimated the amount of variance explained in FMD by BMI and number of MetS components using an incremental sums of squares approach. Distribution data are presented as mean \pm s.D. and outcomes of ANOVA as mean (95\% CI). The alpha level of statistical significance was set at $P<0.05$. Statistical analysis was performed using SPSS for Windows (Version 24.0, SPSS).

\section{Results}

\section{Participant characteristics}

Gender, age and BMI for each of the groups are summarised in Table 1 . The differences between the mean BMI and components of MetS were in line with WHO and IDF classifications, respectively. Age and gender were not significantly different between groups $(P>0.05)$. Overall, habitual physical activity did not differ between BMI categories of MetS; however, sedentary behaviour was greater in both of the obese groups compared to nonobese MetS- $(P \leq 0.028)$ and light intensity PA was lower $(P \leq 0.001)$. Total energy consumption, carbohydrate, protein and fat did not differ significantly between groups $(P>0.05)$ (Table 1). Macronutrient percentages of all groups combined were $53 \pm 10 \%$ carbohydrate, $26 \pm 9 \%$ protein, and $21 \pm 4 \%$ fat.

\section{Flow mediated dilation}

FMD was higher in the MetS- individuals in both the non-obese and obese groups (Fig. 1A). The non-obese MetS- individuals had a greater FMD than their MetS+

Table 1 Descriptive data, mean \pm S.D. of clinical values, physical activity and dietary data of each group categorised for obesity and subsequently MetS.

\begin{tabular}{l}
\hline \\
\hline Gender \\
Males $(n)$ \\
Females $(n)$ \\
Age (years) \\
BMI (kg/m ${ }^{2}$ ) \\
Components of metabolic syndrome \\
Waist circumference (cm) \\
Systolic BP (mmHg) \\
Diastolic BP (mmHg) \\
Fasting glucose (mmol/L) \\
Triglyceride (mmol/L) \\
HDL-cholesterol (mmol/L) \\
Physical activity \\
Energy expenditure (kJ/day) \\
PA duration (>1.5 METS) (min/day) \\
Sedentary (<1.5 METS) (min/day) \\
Light (1.3-3 METS) (min/day) \\
MVPA (>3 METS) (min/day) \\
Dietary analysis \\
Energy intake (kJ/day) \\
Carbohydrate (g/day) \\
Protein (g/day) \\
Fat (g/day) \\
\hline
\end{tabular}

\begin{tabular}{|c|c|}
\hline \multicolumn{2}{|c|}{ Non-obese } \\
\hline MetS- $(n=10)$ & MetS+ $(n=10)$ \\
\hline 9 & 8 \\
\hline 1 & 2 \\
\hline $43 \pm 14$ & $48 \pm 9$ \\
\hline $24.6 \pm 3.1$ & $26.9 \pm 2.0$ \\
\hline $89 \pm 10$ & $97 \pm 6$ \\
\hline $125 \pm 13$ & $143 \pm 11$ \\
\hline $79 \pm 13$ & $95 \pm 15$ \\
\hline $5.0 \pm 0.4$ & $5.4 \pm 0.3$ \\
\hline $1.1 \pm 0.8$ & $1.4 \pm 0.5$ \\
\hline $1.7 \pm 0.4$ & $1.7 \pm 0.7$ \\
\hline $12143 \pm 3641$ & $12226 \pm 1743$ \\
\hline $482 \pm 117$ & $340 \pm 137$ \\
\hline $909 \pm 113^{*}$ & $1027 \pm 91$ \\
\hline $321 \pm 73^{*}$ & $253 \pm 74$ \\
\hline $165 \pm 93$ & $117 \pm 52$ \\
\hline $9532 \pm 2008$ & $8272 \pm 1441$ \\
\hline $206 \pm 79$ & $209 \pm 59$ \\
\hline $95 \pm 16$ & $91 \pm 13$ \\
\hline $92 \pm 24$ & $73 \pm 9$ \\
\hline
\end{tabular}

\begin{tabular}{|c|c|}
\hline \multicolumn{2}{|c|}{ Obese } \\
\hline MetS $-(n=12)$ & MetS $+(n=12)$ \\
\hline 7 & 6 \\
\hline 5 & 6 \\
\hline $43 \pm 14$ & $36 \pm 11$ \\
\hline $33.7 \pm 4.7$ & $33.9 \pm 2.6$ \\
\hline $105 \pm 15$ & $111 \pm 9$ \\
\hline $126 \pm 14$ & $149 \pm 18$ \\
\hline $77 \pm 5$ & $92 \pm 12$ \\
\hline $4.9 \pm 0.4$ & $5.5 \pm 1.1$ \\
\hline $1.2 \pm 0.8$ & $1.8 \pm 1.0$ \\
\hline $1.6 \pm 0.5$ & $1.3 \pm 0.3$ \\
\hline $12079 \pm 3951$ & $13281 \pm 3104$ \\
\hline $304 \pm 160$ & $311 \pm 179$ \\
\hline $1074 \pm 166$ & $1132 \pm 125$ \\
\hline $186 \pm 96$ & $176 \pm 56$ \\
\hline $121 \pm 86$ & $109 \pm 104$ \\
\hline $9629 \pm 2201$ & $8019 \pm 1217$ \\
\hline $214 \pm 76$ & $236 \pm 39$ \\
\hline $130 \pm 54$ & $85 \pm 14$ \\
\hline $95 \pm 26$ & $65 \pm 23$ \\
\hline
\end{tabular}

*significantly different to both obese groups.

BP, blood pressure; F, female; HDL, high-density lipoprotein; M, male; METS, metabolic equivalents; MetS, metabolic syndrome; MVPA, moderatevigorous physical activity; PA, physical activity. 
counterparts $(4.1 \%(1.0,7.3 ; P=0.004))$ and obese MetS+ $(4.3 \%(1.3,7.3 ; P=0.002))$, with no difference compared to obese MetS-. The mean difference between the obese MetS- and obese MetS+ was 6.2\% (3.1, 9.2; $P<0.0001)$, and non-obese MetS+ was 6.0\% (2.8, 9.2; $P<0.0001)$. There was no significant difference between the MetS+ groups. An increased number of MetS components was associated with a lower FMD ( $P=0.04$; Fig. $2 \mathrm{~A})$, differences were observed from the healthy reference group $(0$ components) for those with $3(P=0.005)$ or $\geq 4(P=0.023)$ components of MetS. In contrast, when using a healthy BMI as a reference group (18.5-24.9 $\left.\mathrm{kg} / \mathrm{m}^{2}\right)$, none of the categories were statistically different for FMD $(P=0.27$; Fig. 2B). Furthermore, there was no correlation between BMI and FMD $\left(r^{2}=0.01 ; P=0.512\right.$; Fig. 2 C). The variance of FMD explained, when controlling for age and sex, by BMI was $1.1 \%$ and by MetS was $19.7 \%$. There were negligible and non-statistically significant differences in baseline or peak arterial diameter, shear rate or time to peak between groups $(P>0.05)$. All vascular data are summarised in Table 2 .

\section{Cardio-respiratory fitness (CRF)}

$\dot{\mathrm{V}}_{2}$ peak was greatest in non-obese MetS-, similar in nonobese MetS+ and obese MetS-, and lowest in obese MetS+ (Fig. 1B). Obese MetS+ individuals had a significantly lower CRF than non-obese MetS- by $13.9 \mathrm{~mL} / \mathrm{min} / \mathrm{kg}$ (6.0, 21.7; $P<0.0001)$. Differences between the MetS - groups just fell short of conventional statistical significance $(P=0.056)$. The between-group differences are also consistent when $\dot{\mathrm{V}}_{2}$ peak is expressed relative to lean mass. Interestingly, when FMD was adjusted for individual differences in CRF, the difference in FMD between groups remained and was of similar magnitude $(P<0.05)$.

\section{MRS determination of liver fat}

Group differences in liver fat were non-significant $(P=0.099)$; however, the mean values for each group suggest a trend towards greater levels of liver fat in the MetS+ groups (Fig. 1C).

\section{Assessment of body composition (BIA and MRI)}

$B I A$

Total body fat measured in percentage and mass was significantly lower in the non-obese groups compared to
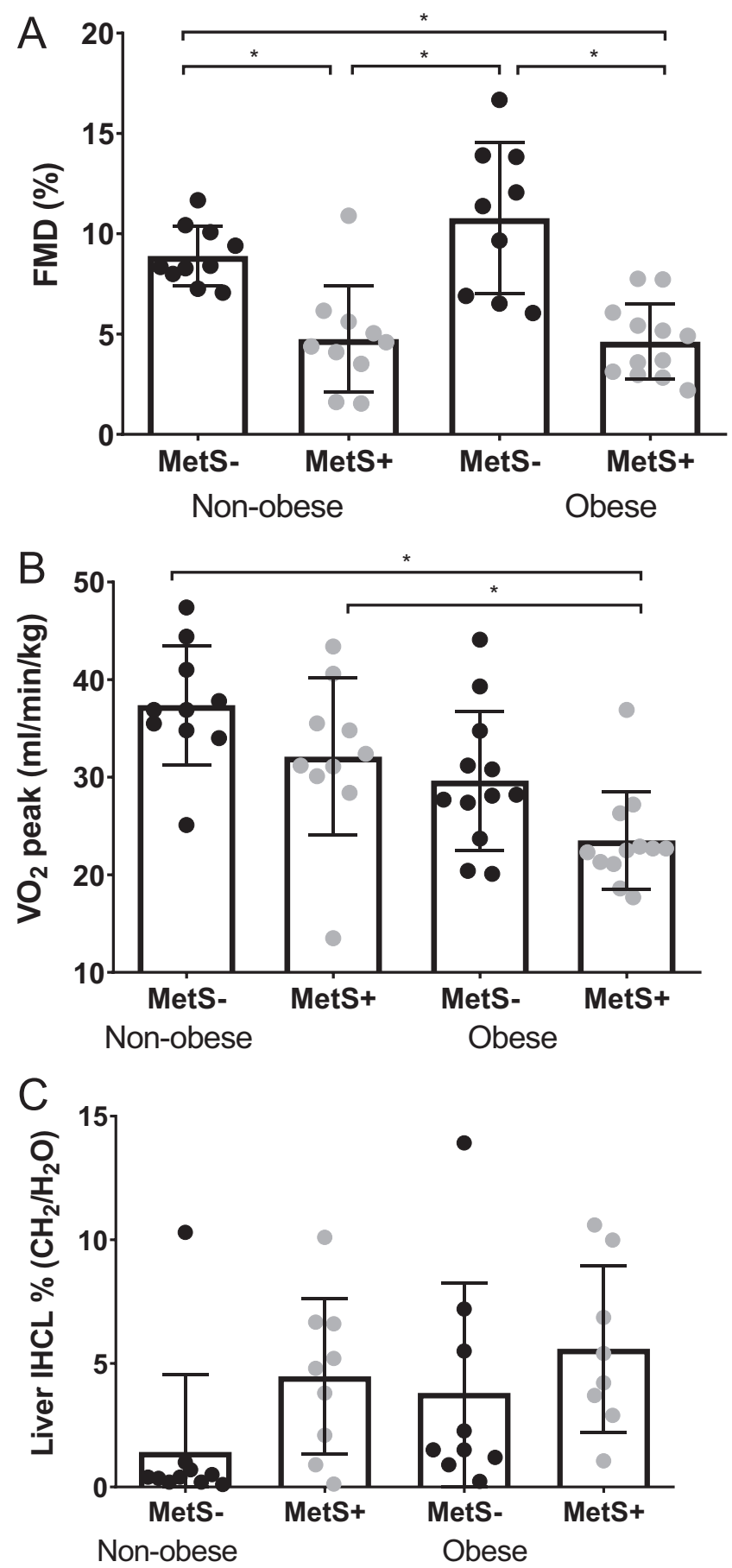

Figure 1

Individual participant plots for (A) flow mediated dilation (FMD), (B) cardio-respiratory fitness ( $\dot{\mathrm{VO}}_{2}$ peak) and (C) 'liver fat' intrahepatic cellular lipid (IHCL) percentage. Black circles, MetS-; grey circles, MetS+; non-obese are grouped left and obese are grouped right. Group mean \pm S.D. data are presented as bar. ${ }^{\star} P<0.05$, group difference. 

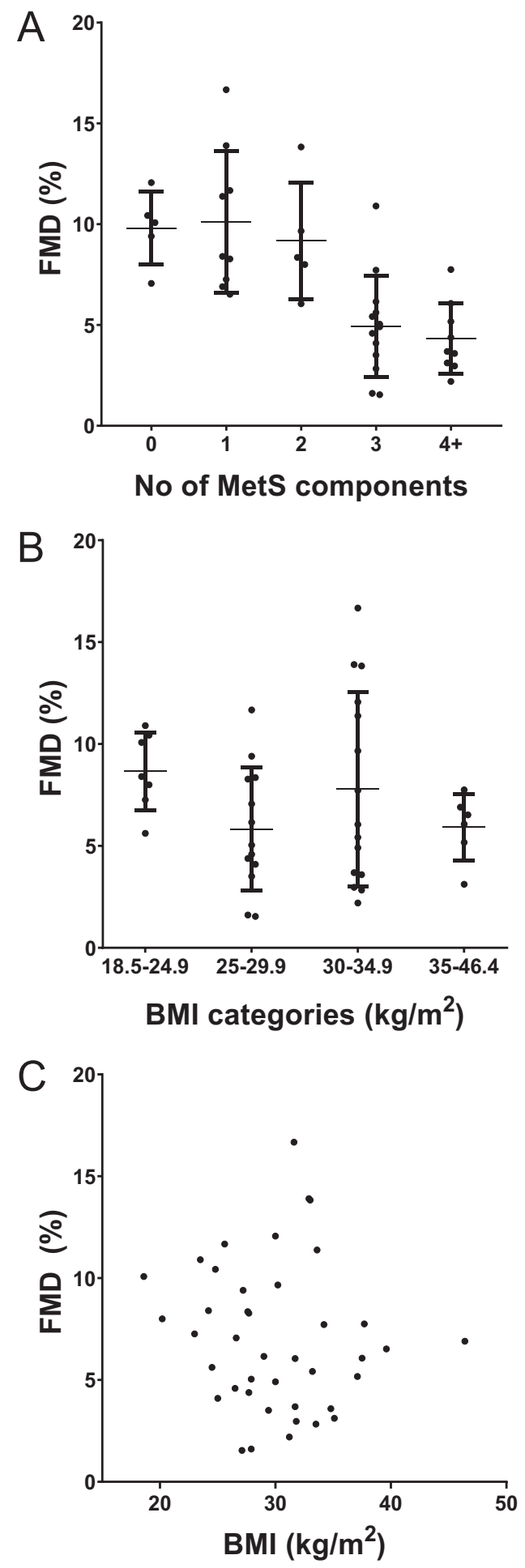

Figure 2

Individual plots for all 44 participants (A) flow mediated dilation (FMD) categorised for number of metabolic syndrome (MetS) components, (B) FMD categorised for (BMI) classifications and $(C)$ showing individual points for flow mediated dilation (FMD) and body mass index (BMI). the obese groups $(P<0.05$; Table 3$)$; however, there were no significant differences between MetS- vs MetS+ within the BMI groups. Visceral fat rating was significantly lower in the non-obese MetS- group $(P<0.05)$ but there were no other significant differences. No significant differences were observed in BIA derived fat free mass or muscle mass between any of the groups.

$M R I$

Total s.c. adipose tissue (SAT) and whole-body fat were significantly lower in the non-obese MetS- than both obese groups $(P<0.05)$. Abdominal SAT was lower in both non-obese groups $(P<0.05)$. Visceral adipose tissue was significantly lower in non-obese MetS- when compared to obese MetS-. Of note, there were no significant differences between MetS- vs MetS+ within the BMI groups but the data was not available for all participants.

\section{Discussion}

The aim of study was to determine to what extent MetS or obesity is associated with endothelial function as a surrogate marker of cardiovascular health. The integration of measures of dietary intake and domains of physical activity, biochemical and anthropometric measures including characterisation of components of MetS (IDF consensus) and body composition using MRI and spectroscopy enabled comprehensive phenotyping of individuals within age- and sex-matched groups. The major finding was that individuals with MetS (i.e. metabolically unhealthy individuals) exhibit endothelial dysfunction (lower FMD), irrespective of their obesity status. In contrast, individuals without MetS (i.e. metabolically healthy individuals) had relatively preserved endothelial function (higher FMD). Convincingly, MetS status is significantly associated with endothelial function whereas BMI is not. Alarmingly, the FMD differences between the metabolic phenotypes in this study (MetS+ vs MetS-) was identified as $\sim 4-6 \%$, with indication towards an increased risk of incident CVD. Our data highlight the association of increased CVD risk in metabolically unhealthy individuals, irrespective of their obesity status, and suggest that preserved metabolic health may indeed confer a degree of cardiovascular protection and attenuate (but not necessarily eliminate) the risks associated with obesity.

Our findings support the existence of distinct phenotypes within different categories of BMI, where 
Table 2 Differences in the brachial artery vascular function between groups categorised for obesity and subsequently MetS.

Data are presented as mean \pm S.D.

\begin{tabular}{l}
\hline \\
\hline Males $(n)$ \\
Females $(n)$ \\
Flow-dediated dilation $(\%)$ \\
Baseline diameter $(\mathrm{mm})$ \\
Peak diameter $(\mathrm{mm})$ \\
Shear rate \\
Tuc $\left(\mathrm{s}^{-1} \times 10^{3}\right)$ \\
Time to peak $(\mathrm{s})$
\end{tabular}

\begin{tabular}{ccc}
\hline \multicolumn{2}{c}{ Non-obese } \\
\cline { 1 - 1 } MetS- $(n=10)$ & MetS $+(n=10)$ \\
\hline 9 & & 8 \\
1 & & 2 \\
$8.6 \pm 1.2$ & & $4.7 \pm 2.6$ \\
$0.42 \pm 0.06$ & & $0.44 \pm 0.06$ \\
$0.46 \pm 0.06$ & & $0.45 \pm 0.06$ \\
$15395 \pm 8421$ & & $11669 \pm 7808$ \\
$44.4 \pm 19.6$ & $32.7 \pm 19.2$ \\
\hline
\end{tabular}

\begin{tabular}{cr}
\hline Obese \\
\hline MetS- $(n=12)$ \\
\hline 7 \\
5 \\
$10.8 \pm 3.6$ \\
$0.40 \pm 0.1$ \\
$0.44 \pm 0.1$ \\
$13048 \pm 23067$ \\
$71.9 \pm 59.1$
\end{tabular}

MetS+ individuals exhibit a cluster of metabolic abnormalities (e.g. insulin resistance, hypertension and dyslipidemia). The data suggests that endothelial dysfunction is not explained by the absolute fat mass, but rather is determined (in part) by associated cardiometabolic dysfunction/risk factors alongside known and so far unidentified factors. Individuals with MetS (nonobese and obese) have an unfavourable cardiovascular profile with a lower FMD (an early marker of atherosclerotic disease), while those without MetS (non-obese and obese) have comparable endothelial function. This phenomenon whereby other measures of cardiovascular function differ between metabolically healthy vs metabolically unhealthy obese adults is observed not only for macrovascular complications, as here and in previous investigations (31), but also for microvascular function (32). Using identical phenotypic classification, we have previously shown similar trends for myocardial systolic and diastolic dysfunction (measured by tissue doppler imaging with transthoracic echocardiography). We observed impaired myocardial performance related to poor metabolic health but not related to levels of fat mass or to differing amounts of ectopic fat stores (visceral and liver) (33). Mechanisms such as inflammation, increased circulating free fatty acids and pro-inflammatory cytokines have been proposed as mediators of this impact on cardiovascular risk (34).

The increasing interest in the study of differing metabolic phenotypes has led many to investigate putative behavioural determinants (e.g. physical activity, diet); however, findings remain equivocal (35). We found no difference between the groups for PA (even when domains of physical activity were analysed) or in their total energy intake/macronutrient intake. We note the disparity between energy intake and expenditure, ostensibly showing the participants in a negative energy balance; however, we recognise that energy intake is largely underreported, particularly in obese adults. Dietary assessment was not a primary outcome variable and was assessed using the best resources available. Cardiorespiratory fitness was highest in the healthy reference group (non-obese

Table 3 Body composition data, mean \pm S.D. derived from both bioelectrical impedance and MRI quantification presented for each group categorised for obesity and subsequently MetS.

\begin{tabular}{|c|c|c|c|c|}
\hline & & & & \\
\hline & MetS- & MetS+ & MetS- & MetS+ \\
\hline Bioelectrical impedance quar & & & & \\
\hline$n$ & 10 & 10 & 12 & 12 \\
\hline Fat $(\%)$ & $21.5 \pm 5.6^{*}$ & $27.5 \pm 5.1 *$ & $39.4 \pm 7.0$ & $39.4 \pm 7.8$ \\
\hline Fat mass (kg) & $16.4 \pm 5.5^{*}$ & $22.3 \pm 3.4^{*}$ & $38.1 \pm 10.9$ & $39.1 \pm 8.2$ \\
\hline Fat free mass (kg) & $58.5 \pm 8.0$ & $59.2 \pm 8.0$ & $58.4 \pm 11.5$ & $60.9 \pm 12.9$ \\
\hline Muscle mass (kg) & $55.5 \pm 7.7$ & $56.2 \pm 7.6$ & $55.4 \pm 7.7$ & $57.9 \pm 12.2$ \\
\hline Visceral fat rating & $8 \pm 3^{*}$ & $10 \pm 3$ & $13 \pm 5$ & $14 \pm 5$ \\
\hline MRI quantification & & & & \\
\hline$n$ & 10 & 8 & 7 & 7 \\
\hline Total SAT (L) & $15.3 \pm 3.8^{*}$ & $17.9 \pm 4.4$ & $29.9 \pm 11.9$ & $28.6 \pm 13.1$ \\
\hline Abdominal SAT (L) & $3.9 \pm 1.8 * *$ & $5.6 \pm 1.1 * *$ & $9.7 \pm 4.2$ & $12.9 \pm 7.7$ \\
\hline Visceral adipose tissue $(\mathrm{L})$ & $3.0 \pm 1.9 * \star \star$ & $4.6 \pm 1.7$ & $6.0 \pm 2.3$ & $5.5 \pm 2.1$ \\
\hline Internal fat $(\mathrm{L})$ & $5.7 \pm 2.6$ & $8.1 \pm 3.4$ & $9.9 \pm 3.6$ & $9.3 \pm 2.9$ \\
\hline Whole-body fat $(\mathrm{L})$ & $21.0 \pm 5.3^{*}$ & $26.0 \pm 2.6^{*}$ & $39.7 \pm 12.7$ & $40.7 \pm 10.4$ \\
\hline
\end{tabular}


MetS-) and lowest in the obese MetS+ group perhaps as expected, although interestingly both groups of nonobese adults and obese MetS- had comparable fitness. A higher cardiorespiratory fitness is typically associated with a better metabolic profile and reduced CVD risk (36), and our data support this. In the MetS- obese group, we observed FMD 15\%, this data is somewhat striking but not abnormal. While obesity has many comorbidities, the role of fitness is also recognised as an important prognostic marker that differs across phenotypes (37) and some researchers suggest that recommendations to reduce mortality risk should focus on increasing fitness rather than on weight loss (38). Although we interpret this data with caution, it is reasonable to suggest that intrinsic biological mechanisms may contribute to the differences we observe in these phenotypes (such as subacute inflammation, levels of oxidative stress, levels of different regulatory miRNA and adiponectin (39)).

Many authors suggest that cross-sectional observations of preserved metabolic health in obese adults likely represent a transient phenomenon and question their clinical utility and significance. Longitudinal studies are needed to address these important questions. One such study found that $50 \%$ of healthy obese progressed to an unhealthy metabolic status over a 10-year follow-up period (40). Interpretation of such studies is hampered by the lack of an agreed definition of 'metabolically healthy' (41); conclusions about the degree of protection against $\mathrm{CV}$ disease and T2D will clearly depend on the criteria of metabolic health. We opted for the IDF classification of MetS, as the most recent and internationally harmonised definition. Furthermore, FMD is often (as here) studied in the fasted state, yet humans spend a significant of their time in a post-prandial state. Examination of post-prandial endothelial function between the phenotypes described in this manuscript maybe warranted and highlight more profound differences. In particular, the post-prandial state following consumption of a high-fat meal may be associated with oxidative stress and inflammation, which are potentially important mediators of impaired postprandial vascular function and may differ between these individuals.

We acknowledge limitations of the current study, including a relatively small sample size and its crosssectional design. Participants were recruited via local advertisement, which limits external validity as this yielded only white Europeans; defining a causal relationship with validity at a global population level is therefore not possible. However, we believe the study has significant merit. The study was powered to detect meaningful differences in the primary outcome measure (FMD). It should be acknowledged that there are outliers (Fig. 2C), but that removal of these data does not alter the outcome of statistical analyses, so the decision was made to include the data set in its entirety. It utilises objective monitoring of physical activity, a gold standard measurement of cardio-respiratory fitness combined with assessment of body composition including regional (VAT/SAT) and tissue specific (liver) fat and a novel prognostic marker for cardiovascular health, that of endothelial function. Liver fat was not our primary outcome and thus the study was not adequately powered for this outcome. Importantly, this measure was utilised to comprehensively phenotype the individuals. Based on previous work regarding fat deposition, we expected a greater propensity to deposit fat within the liver in the metabolically unhealthy (MetS+) phenotypes. This propensity was observed but did not reach statistical significance between groups. While the present results demonstrate that endothelial function is impaired in those with MetS, larger studies are required with a follow-up design to determine measured cardiovascular function rather than predicted CVD. This has been undertaken to a limited extent in a multi-ethnic population study but did not include the classification of sub-phenotypes (42).

In conclusion, the current study provides evidence for impaired NO-mediated endothelial function in both non-obese and obese individuals who have multiple components of MetS (with comparable cardiovascular function in adults without MetS regardless of obesity status). Considering the definition of obesity as a disease (WHO) that recognises the impact of excessive fat accumulation on end-organ complications and the need to triage medical resources to those most in need, earlier detection and more focussed interventions in metabolically unhealthy individuals should be a priority rather than using a purely BMI-centric approach.

\footnotetext{
Declaration of interest

The authors declare that there is no conflict of interest that could be perceived as prejudicing the impartiality of this study.
}

\section{Funding}

This work was supported by Diabetes UK (grant number 13/0004719), with additional funding support from the MRC-Arthritis Research UK Centre for Integrated research into Musculoskeletal Ageing (CIMA), Institute of Ageing and Chronic Disease, University of Liverpool. 


\section{Acknowledgements}

The authors would like to acknowledge Mr Andrew Irwin (Obesity and Endocrinology, Liverpool University Hospital NHS Foundation Trust, Liverpool, UK) for clinical assistance and Ms Val Adams for radiographic expertise at LiMRIC. Further to Ms Olivia Pepper, Mr Daniel Watkin and Mr Rory O'Dolan for their assistance during data collection.

\section{References}

1 Berrington de Gonzalez A, Hartge P, Cerhan JR, Flint AJ, Hannan L, MacInnis RJ, Moore SC, Tobias GS, Anton-Culver H, Freeman LB et al. Body-mass index and mortality among 1.46 million white adults. New England Journal of Medicine 2010363 2211-2219. (https://doi. org/10.1056/NEJMoa1000367)

2 Flegal KM, Kit BK, Orpana H \& Graubard BI. Association of all-cause mortality with overweight and obesity using standard body mass index categories: a systematic review and meta-analysis. JAMA 2013 309 71-82. (https://doi.org/10.1001/jama.2012.113905)

3 Troiano RP, Frongillo Jr EA, Sobal J \& Levitsky DA. The relationship between body weight and mortality: a quantitative analysis of combined information from existing studies. International Journal of Obesity and Related Metabolic Disorders 199620 63-75.

4 Carnethon MR, Rasmussen-Torvik LJ \& Palaniappan L. The obesity paradox in diabetes. Current Cardiology Reports 201416 446. (https:// doi.org/10.1007/s11886-013-0446-3)

5 Lavie CJ, Milani RV \& Ventura HO. Obesity and cardiovascular disease: risk factor, paradox, and impact of weight loss. Journal of the American College of Cardiology 200953 1925-1932. (https://doi. org/10.1016/j.jacc.2008.12.068)

6 Alberti KG, Eckel RH, Grundy SM, Zimmet PZ, Cleeman JI, Donato KA, Fruchart JC, James WP, Loria CM, Smith Jr SC et al. Harmonizing the metabolic syndrome: a joint interim statement of the International Diabetes Federation Task Force on Epidemiology and Prevention; National Heart, Lung, and Blood Institute; American Heart Association; World Heart Federation; International Atherosclerosis Society; and International Association for the Study of Obesity. Circulation 2009120 1640-1645. (https://doi. org/10.1161/CIRCULATIONAHA.109.192644)

7 Caleyachetty R, Thomas GN, Toulis KA, Mohammed N, Gokhale KM, Balachandran K \& Nirantharakumar K. Metabolically healthy obese and incident cardiovascular disease events among 3.5 million men and women. Journal of the American College of Cardiology $2017 \mathbf{7 0}$ 1429-1437. (https://doi.org/10.1016/j.jacc.2017.07.763)

8 Phillips CM. Metabolically healthy obesity across the life course: epidemiology, determinants, and implications. Annals of the New York Academy of Sciences 20171391 85-100. (https://doi.org/10.1111/ nyas.13230)

9 Stefan N, Schick F \& Häring HU. Causes, characteristics, and consequences of metabolically unhealthy normal weight in humans. Cell Metabolism 201726 292-300. (https://doi.org/10.1016/j. cmet.2017.07.008)

10 Eckel N, Li Y, Kuxhaus O, Stefan N, Hu FB \& Schulze MB. Transition from metabolic healthy to unhealthy phenotypes and association with cardiovascular disease risk across BMI categories in 90257 women (the Nurses' Health Study): 30 year follow-up from a prospective cohort study. Lancet: Diabetes and Endocrinology 20186 714-724. (https://doi.org/10.1016/S2213-8587(18)30137-2)

11 Khan SS, Ning H, Wilkins JT, Allen N, Carnethon M, Berry JD, Sweis RN \& Lloyd-Jones DM. Association of body mass index with lifetime risk of cardiovascular disease and compression of Morbidity Association. JAMA Cardiology 20183 280-287. (https://doi. org/10.1001/jamacardio.2018.0022)

12 Morkedal B, Vatten LJ, Romundstad PR, Laugsand LE \& Janszky I. Risk of myocardial infarction and heart failure among metabolically healthy but obese individuals: HUNT (Nord-Trondelag Health Study), Norway. Journal of the American College of Cardiology 201463 1071-1078. (https://doi.org/10.1016/j.jacc.2013.11.035)

13 Hamer M \& Stamatakis E. Metabolically healthy obesity and risk of all-cause and cardiovascular disease mortality. Journal of Clinical Endocrinology and Metabolism 201297 2482-2488. (https://doi. org/10.1210/jc.2011-3475)

14 Mongraw-Chaffin M, Foster MC, Anderson CAM, Burke GL, Haq N, Kalyani RR, Ouyang P, Sibley CT, Tracy R, Woodward M et al. Metabolically healthy obesity, transition to metabolic syndrome, and cardiovascular risk. Journal of the American College of Cardiology 2018 71 1857-1865. (https://doi.org/10.1016/j.jacc.2018.02.055)

15 Furchgott RF \& Zawadzki JV. The obligatory role of endothelial cells in the relaxation of arterial smooth muscle by acetylcholine. Nature 1980288 373-376. (https://doi.org/10.1038/288373a0)

16 Green DJ, Jones H, Thijssen D, Cable NT \& Atkinson G. Flowmediated dilation and cardiovascular event prediction: does nitric oxide matter? Hypertension 201157 363-369. (https://doi. org/10.1161/HYPERTENSIONAHA.110.167015)

17 Flammer AJ, Anderson T, Celermajer DS, Creager MA, Deanfield J, Ganz P, Hamburg NM, Lüscher TF, Shechter M, Taddei S et al. The assessment of endothelial function: from research into clinical practice. Circulation 2012126 753-767. (https://doi.org/10.1161/ CIRCULATIONAHA.112.093245)

18 Widlansky ME, Gokce N, Keaney Jr JF \& Vita JA. The clinical implications of endothelial dysfunction. Journal of the American College of Cardiology 200342 1149-1160. (https://doi.org/10.1016/ s0735-1097(03)00994-x)

19 De Boer MP, Meijer RI, Wijnstok NJ, Jonk AM, Houben AJ, Stehouwer CD, Smulders YM, Eringa EC \& Serné EH. Microvascular dysfunction: a potential mechanism in the pathogenesis of obesityassociated insulin resistance and hypertension. Microcirculation 2012 19 5-18. (https://doi.org/10.1111/j.1549-8719.2011.00130.x)

20 Jonk AM, Houben AJ, de Jongh RT, Serne EH, Schaper NC \& Stehouwer CD. Microvascular dysfunction in obesity: a potential mechanism in the pathogenesis of obesity-associated insulin resistance and hypertension. Physiology 200722 252-260. (https:// doi.org/10.1152/physiol.00012.2007)

21 Inaba Y, Chen JA \& Bergmann SR. Prediction of future cardiovascular outcomes by flow-mediated vasodilatation of brachial artery: a meta-analysis. International Journal of Cardiovascular Imaging 201026 631-640. (https://doi.org/10.1007/s10554-010-9616-1)

22 Sprung VS, Jones H, Pugh CJ, Aziz NF, Daousi C, Kemp GJ, Green DJ, Cable NT \& Cuthbertson DJ. Endothelial dysfunction in hyperandrogenic polycystic ovary syndrome is not explained by either obesity or ectopic fat deposition. Clinical Science 2014126 67-74. (https://doi.org/10.1042/CS20130186)

23 Cuthbertson DJ, Shojaee-Moradie F, Sprung VS, Jones H, Pugh CJ, Richardson P, Kemp GJ, Barrett M, Jackson NC, Thomas EL et al. Dissociation between exercise-induced reduction in liver fat and changes in hepatic and peripheral glucose homeostasis in obese patients with non-alcoholic fatty liver disease. Clinical Science 2016 130 93-104. (https://doi.org/10.1042/CS20150447)

24 Cuthbertson DJ, Irwin A, Gardner CJ, Daousi C, Purewal T, Furlong N, Goenka N, Thomas EL, Adams VL, Pushpakom SP et al. Improved glycaemia correlates with liver fat reduction in obese, type 2 diabetes, patients given glucagon-like peptide-1 (GLP-1) receptor agonists. PLoS ONE 20127 e50117. (https://doi.org/10.1371/journal. pone.0050117)

25 Todd CS, Jones HE, Garber TC, Afnan-Holmes H, Woolgar H, Bekker LG \& Myer L. Awareness and interest in intrauterine contraceptive device use among HIV-positive women in Cape Town, South Africa. Infectious Diseases in Obstetrics and Gynecology 2012 2012 956145. (https://doi.org/10.1155/2012/956145)

26 Johannsen DL, Calabro MA, Stewart J, Franke W, Rood JC \& Welk GJ. Accuracy of armband monitors for measuring daily 
energy expenditure in healthy adults. Medicine and Science in Sports and Exercise 201042 2134-2140. (https://doi.org/10.1249/ MSS.0b013e3181e0b3ff)

27 Scheers T, Philippaerts R \& Lefevre J. Variability in physical activity patterns as measured by the SenseWear Armband: how many days are needed? European Journal of Applied Physiology 2012112 1653-1662. (https://doi.org/10.1007/s00421-011-2131-9)

28 Faul F, Erdfelder E, Buchner A \& Lang AG. Statistical power analyses using $\mathrm{G}^{*}$ Power 3.1: tests for correlation and regression analyses. Behavior Research Methods 200941 1149-1160. (https://doi. org/10.3758/BRM.41.4.1149)

29 Atkinson G, Batterham AM, Thijssen DH \& Green DJ. A new approach to improve the specificity of flow-mediated dilation for indicating endothelial function in cardiovascular research. Journal of Hypertension 201331 287-291. (https://doi.org/10.1097/ HJH.0b013e32835b8164)

30 Atkinson G \& Batterham AM. Allometric scaling of diameter change in the original flow-mediated dilation protocol. Atherosclerosis 2013226 425-427. (https://doi.org/10.1016/j. atherosclerosis.2012.11.027)

31 Khan UI, Wang D, Thurston RC, Sowers M, Sutton-Tyrrell K, Matthews KA, Barinas-Mitchell E \& Wildman RP. Burden of subclinical cardiovascular disease in 'metabolically benign' and 'at-risk' overweight and obese women: the Study of Women's Health Across the Nation (SWAN). Atherosclerosis 2011217 179-186. (https://doi.org/10.1016/j.atherosclerosis.2011.01.007)

32 Brant LC, Wang N, Ojeda FM, LaValley M, Barreto SM, Benjamin EJ, Mitchell GF, Vasan RS, Palmisano JN, Münzel T et al. Relations of metabolically healthy and unhealthy obesity to digital vascular function in three community-based cohorts: a meta-analysis. Journal of the American Heart Association 20176 e004199. (https://doi. org/10.1161/JAHA.116.004199)

33 Dobson R, Burgess MI, Sprung VS, Irwin A, Hamer M, Jones J, Daousi C, Adams V, Kemp GJ, Shojaee-Moradie F et al. Metabolically healthy and unhealthy obesity: differential effects on myocardial function according to metabolic syndrome, rather than obesity. International Journal of Obesity 201640 153-161. (https://doi. org/10.1038/ijo.2015.151)
34 Panagiotakos DB, Pitsavos C, Yannakoulia M, Chrysohoou C \& Stefanadis C. The implication of obesity and central fat on markers of chronic inflammation: the Attica study. Atherosclerosis 2005183 308-315. (https://doi.org/10.1016/j.atherosclerosis.2005.03.010)

35 Phillips CM, Dillon C, Harrington JM, McCarthy VJ, Kearney PM, Fitzgerald AP \& Perry IJ. Defining metabolically healthy obesity: role of dietary and lifestyle factors. PLOS ONE 20138 e76188. (https://doi. org/10.1371/journal.pone.0076188)

36 Myers J, McAuley P, Lavie CJ, Despres JP, Arena R \& Kokkinos P. Physical activity and cardiorespiratory fitness as major markers of cardiovascular risk: their independent and interwoven importance to health status. Progress in Cardiovascular Diseases 201557 306-314. (https://doi.org/10.1016/j.pcad.2014.09.011)

37 Ortega FB, Cadenas-Sanchez C, Sui X, Blair SN \& Lavie CJ. Role of fitness in the metabolically healthy but obese phenotype: a review and update. Progress in Cardiovascular Diseases 201558 76-86. (https://doi.org/10.1016/j.pcad.2015.05.001)

38 Barry VW, Baruth M, Beets MW, Durstine JL, Liu J \& Blair SN. Fitness vs. fatness on all-cause mortality: a meta-analysis. Progress in Cardiovascular Diseases 201456 382-390. (https://doi.org/10.1016/j. pcad.2013.09.002)

39 Muñoz-Garach A, Cornejo-Pareja I \& Tinahones FJ. Does metabolically healthy obesity exist? Nutrients 20168320 . (https:// doi.org/10.3390/nu8060320)

40 Kouvari M, Panagiotakos DB, Yannakoulia M, Georgousopoulou E, Critselis E, Chrysohoou C, Tousoulis D \& Pitsavos C. Transition from metabolically benign to metabolically unhealthy obesity and 10-year cardiovascular disease incidence: the Attica cohort study. Metabolism 201993 18-24. (https://doi.org/10.1016/j.metabol.2019.01.003)

41 Phillips CM. Metabolically healthy obesity: definitions, determinants and clinical implications. Reviews in Endocrine and Metabolic Disorders 201314 219-227. (https://doi.org/10.1007/s11154-013-9252-x)

42 Yeboah J, Folsom AR, Burke GL, Johnson C, Polak JF, Post W, Lima JA, Crouse JR \& Herrington DM. Predictive value of brachial flow-mediated dilation for incident cardiovascular events in a population-based study: the multi-ethnic study of atherosclerosis. Circulation 2009120 502-509. (https://doi.org/10.1161/ CIRCULATIONAHA.109.864801)

Received 5 February 2020

Revised version received 17 April 2020

Accepted 26 May 2020 